\title{
Development and validation of a high performance liquid chromatographic-mass spectrometry method for the simultaneous quantification of 10 trichothecenes in ultra-high temperature processed cow milk
}

Myra E. Flores-Flores, Elena González-Peñas*.

Organic and Pharmaceutical Chemistry Department, Faculty of Pharmacy, University of Navarra. C/ Irunlarrea 1, 31008, Pamplona, Navarra, Spain.

*Corresponding author: Elena González-Peñas PhD: Tel: +34 948 425653. Fax: +34

948 425652. E-mail: mgpenas@unav.es

E-mail addresses of all coauthors: Myra E. Flores-Flores: mflores.6@alumni.unav.es

\begin{abstract}
An LC-MS/MS (QqQ) method has been developed and validated for simultaneous determination of the following trichothecenes in UHT cow milk: nivalenol (NIV), deoxynivalenol (DON), deepoxy-deoxynivalenol (DOM-1), 3-acetyldeoxynivalenol (3ADON), 15-acetyldeoxynivalenol (15-ADON), neosolaniol (NEO), diacetoxyscirpenol (DAS), fusarenon X (FUS-X), T-2 and HT-2 toxins. Sample treatment is simple and based on the extraction with acetonitrile (ACN), acidified with $0.2 \%$ formic acid, followed by a purification process, adding sodium acetate to the ACN/water extract in order to separate aqueous phase and, consequently, polar components of the milk. Validation of the method for all the 10 mycotoxins was successful; validation parameters taken into account were as follows: limits of detection (LOD) and quantification (LOQ), linearity, precision (within-day and between-day variability), recovery, matrix effect and stability. The LODs were 10.1, 2.5, 1.5, 1.9, 0.1, 0.5, 1.0, 0.08, 0.4 and $0.05 \mathrm{ng} / \mathrm{mL}$ for NIV, DON, DOM-1, FUS-X, NEO, 3-ADON, 15-ADON, DAS, HT-2 and T-2, respectively. Mean recovery values (obtained in intermediate precision conditions) were between 63.5 and $75.8\left(\mathrm{RSD}_{\mathrm{R}} \leq 15 \%\right)$ for all the mycotoxins. All the mycotoxins suffered from matrix effects, especially DON.
\end{abstract}

\section{Keywords}

Mycotoxin; Trichothecenes; Milk; UHT cow milk; LC-MS/MS. 


\section{Introduction}

Mycotoxins are secondary metabolites produced by molds and they can contaminate agricultural commodities intended for human food and animal feed [1]. In fact, approximately $20 \%$ of food products, mainly of plant origin, are contaminated with mycotoxins [2]. The presence of the mycotoxins in human food and animal feed could be a risk to human and animal health due to their toxicity [3] and could also produce economic losses due to refusal of contaminated products, reduced animal production and veterinary costs [4]. Co-occurrence of mycotoxins in food and feed is likely to appear because one type of fungus can produce different mycotoxins, and on the same substrate more than one type of mold could be present.

The Fusarium genus is probably the major producer of mycotoxins on temperate areas [5]. Trichothecenes are among the most frequently detected fusariotoxins in human and animal cereal-based food [6]. Trichothecenes are classified into two groups, A and B, according to their structural characteristics. Type A, including diacetoxyscirpenol (DAS), neosolaniol (NEO), HT-2 and T-2 toxins, can cause vomiting, diarrhea, leukopenia, necrotic lesions and hemorrhage. T-2 toxin is a highly toxic compound, especially for the immune system. It has been related to the Alimentary Toxic Aleukia (ATA) illness [7], to the inhibition of protein synthesis [5], to bovine infertility and to abortion [8]. The European Food Safety Authority [9] considers T-2 toxin to be one of the most dangerous contaminants, and the European Commission has recommended a tolerable daily intake for humans (TDI) of $100 \mathrm{ng} / \mathrm{kg}$ body weight (b.w.) for the sum of T-2 and HT-2 toxins [10].

With regard to type $\mathrm{B}$, the trichothecenes classified into this group are as follows: deoxynivalenol (DON, vomitoxin), 3-acetyldeoxynivalenol (3-ADON), 15acetyldeoxynivalenol (15-ADON), nivalenol (NIV) and fusarenon X (FUS-X). Their toxic effects are food refusal and vomiting, kidney problems and immunosuppression [11]. DON has emetic properties and provokes feed refusal in animal; in addition, it supposedly suppresses resistance to bacterial infections such as Listeria and Salmonella [12]. The European Commission has recommended a TDI of $1.2 \mu \mathrm{g} / \mathrm{kg}$ b.w. per day for NIV[13] and a provisional maximum tolerable daily intake (PMTDI) of $1 \mu \mathrm{g} / \mathrm{kg} \mathrm{b}$.w. for DON and its acetylated derivatives (3-ADON and 15-ADON) [14]. 
Milk is a highly consumed food. For instance, in Europe, more than $150 \mathrm{~kg} / \mathrm{capita} / \mathrm{year}$ are consumed [15]. In 2013, the estimated milk production was approximately 159 million tons per year, approximately $15 \%$ of the European agricultural output [16]. Due to its economic impact and its importance as a human food, especially during the first years of life, it is very important to determine the presence of contaminants in this matrix in order to expand our knowledge regarding this aspect.

There is little information regarding the presence of trichothecenes in milk due to the low number of samples analyzed and their presence in this matrix has not been regulated. Ruminants are supposedly less affected for mycotoxins due to the rumen metabolism [4], and their concentration levels, if any, in milk from healthy cows are supposed to be low. However, certain cow diseases can alter rumen metabolism [17] and favors the possible presence of mycotoxins in milk. Sørensen and Elbæk detected DOM-1 in a $25 \%$ of milk samples obtained from cows with undiagnosed symptoms of disease [18].

In addition, high levels of mycotoxins in feed could also alter rumen metabolism [17]. Some trichothecenes in food intended for animal feeding are a major concern, especially DON, T-2 and HT-2. The European Union has established guidance values for DON in products intended for animal feed and has recommended the collection of more data for T-2 and HT-2 in these matrices. In order to assess co-occurrence, simultaneous analysis of diverse mycotoxins in feed has also been recommended [19].

Few studies regarding transference of trichothecenes into milk have been carried out. Only transmission of DON and T-2 to cow milk has been studied, but in few animals and with variable results due to the use of different doses or routes of administration. DON is mostly biotransformed within the rumen and detected in milk as DON and deepoxy-deoxynivalenol (DOM-1) [20-22], whereas T-2 has been detected in the milk without metabolization [23] and metabolized into HT-2, NEO, 4-deacetylneosolaniol, and 4 more unknown metabolites [24]. Metabolites could have different toxic effects than the parent mycotoxin [25,26]. More studies are needed in order to increase knowledge regarding this aspect. 
Nowadays, mycotoxin detection in milk generally focuses on aflatoxin M1 (AFM1); little is known regarding the presence of other mycotoxins in this matrix. However, our previous survey [27] has found that low levels of other mycotoxins such as DOM-1 can be present in milk samples worldwide. Interestingly, Slovakia and Czech Republic have established maximum limits for mycotoxins other than AFM1 in milk [28]. In the case of different trichothecenes being present in milk, together they could have additive or synergic toxic effects on human health; however, this aspect has not been studied.

In addition, the study of the presence of mycotoxins in milk can be used as a control for animal feed contamination and as a tool to study absorption and distribution of mycotoxins in animals [29].

Validated analytical methods, especially those capable of simultaneous analysis, are needed in order to assure that contaminated milk does not reach consumers; in addition, they will be helpful in broadening our knowledge regarding toxicity, transmission from feed into milk, etc. However, due to the different physicochemical characteristics of mycotoxins and to the complexity of milk composition (lipids, proteins and sugars, among other components), the development of this type of methods is an analytical challenge.

Trichothecenes in milk have usually been analyzed using gas chromatography, in which case a step of derivatization is necessary before the chromatographic analysis. Most of these studies are based on the analysis of a single mycotoxin (usually DON or T-2) instead of the simultaneous analysis of type-A and type-B trichothecenes [18]. The use of liquid chromatography coupled with MS, in which derivatization is not necessary and in which the simultaneous analysis of different chemical structures is permitted, is an advantage. Moreover, LC-MS reaches the high selectivity and sensitivity needed in the determination of contaminants in food matrixes. In fact, methods for multimycotoxin detection in food using this technique have increased over the last years. However, methods devoted to trichothecenes determination in cow milk using LC-MS/MS are scarce.

Jia et al. validated an analytical method for the simultaneous analysis of 58 mycotoxins, including 9 trichothecenes, in commercial milk using UHPLC/ESI Q-Orbitrap and the 
QuEChERS extraction procedure [29]. On the other hand, after acetonitrile and hexane extraction from milk, a clean-up phase by solid phase extraction (SPE), and with the use of LC-ESI-MS/MS, Sørensen and Elbæk simultaneously determined the following trichothecenes: DON, DOM-1, 3-ADON, 15-ADON, DAS, T-2 and HT-2 [18]. In addition, Beltrán et al. determined 18 mycotoxins (nine trichothecenes) in different matrixes, including milk, using UHPLC-MS/MS [30]. Finally, Tsiplakou et al. developed a method capable of determining 11 mycotoxins in milk using LC-MS/MS, including the trichothecenes DAS, T2 and HT-2 [31].

The aim of this work is to develop and to validate a method capable of analyzing 10 trichothecenes (NIV, DON, DOM-1, 13-ADON, 15-ADON, NEO, DAS, FUS-X, HT-2 and T-2) simultaneously in ultra-high temperature (UHT)-cow milk using LC-MS/MS triple quadrupole (QqQ) mass spectrometer. Other available methods for trichothecenes determination in milk use different equipment or include a lower number of trichothecenes in a single analysis. Due to milk composition characteristics, the extraction procedure has been carefully studied, using different extraction solvent mixtures. The validated method was applied to the simultaneous analysis of 10 trichothecenes (type-A and type-B) in 13 samples of UHT cow milk collected in Navarra (Spain).

\section{Experimental}

\subsection{Chemicals and reagents}

Methanol (LC/MS grade), formic acid (mass spectrometry grade, purity > 98\%), ammonium formate (analytical grade), sodium acetate (anhydrous, HPLC grade $>99.0 \%$ ), sodium chloride (ACS reagent >99.0\%) and magnesium sulfate (anhydrous reagent plus >99.5\%) were purchased from Sigma-Aldrich (USA). Deionized water (>18 $\mathrm{M} \Omega \mathrm{cm}^{-1}$ resistivity) was purified using Ultramatic Type I system (ultrapure reagent grade water) from Wasserlab (Spain).

\subsection{Mycotoxin standard solutions}

All mycotoxins (reference material purity $\geq 98 \%$ ) were supplied by Sigma-Aldrich (USA) and kept at $-20^{\circ} \mathrm{C}$. Trichothecenes: nivalenol, deoxynivalenol, deepoxydeoxynivalenol, 3-acetyldeoxynivalenol, 15-acetyldeoxynivalenol, neosolaniol, 
diacetoxyscirpenol, fusarenon $\mathrm{X}, \mathrm{T}-2$ and HT-2 toxins were purchased as standard solutions of $100 \mu \mathrm{g} / \mathrm{mL}$ in acetonitrile.

Ten milliliters of a mixed stock solution in acetonitrile was prepared by diluting appropriate volumes of the individual standard solutions. Before storing at $-20^{\circ} \mathrm{C}$, the mixed stock solution was aliquoted $(1 \mathrm{~mL})$ into microcentrifuge tubes. Each tube was maintained at room temperature and in darkness for $30 \mathrm{~min}$ prior to use. The calculated concentration of each mycotoxin in the mixed stock solution (in $\mathrm{ng} / \mathrm{mL}$ ) was as follows: NIV 1011.4, DON 251.3, DOM-1 151.5, FUS-X 185.0, NEO 10.0, 3-ADON 50.2, 15 ADON 101.1, DAS 8.0, HT-2 40.1 and T-2 5.0. The addition of different volumes of this mixed stock solution to milk allowed the obtainment of calibration samples containing all the analytes in the concentration range desired for each one of them.

\subsection{Safety precautions}

Due to their toxicity, mycotoxins have always been handled in solution, thereby avoiding the formation of dust and aerosols. Face shield and gloves were used during the dilution of mycotoxins and when handling spiked samples. In order to protect mycotoxins from photodegradation all the mycotoxins solutions, samples and spiked samples, were handled under low-light conditions.

\subsection{Instrumentation and analytical conditions}

Chromatographic separation was achieved using an LC system from Agilent Technologies, 1200 series (Germany): degasser (G1379B), binary pump (G1312B), autosampler (G1367C) with thermostat (G1330B) and a thermostatic column compartment (G1316B) equipped with an Ascentis Express C18, $2.7 \mu \mathrm{m}$ particle size, $150 \mathrm{~mm}$ x $2.1 \mathrm{~mm}$ column (Supelco Analytical, USA), maintained at $45^{\circ} \mathrm{C}$. Mobile phase consisted in a mixture of component A $(5 \mathrm{mM}$ ammonium formate and $0.1 \%$ formic acid in water) and B (5mM ammonium formate and $0.1 \%$ formic acid in 95:5 methanol:water). Chromatographic separation was achieved using the following elution program: $0 \mathrm{~min}, 5 \% \mathrm{~B}$, to $28 \% \mathrm{~B}$ at $5.0 \mathrm{~min}$, to $45 \% \mathrm{~B}$ at $10.5 \mathrm{~min}$, to $60 \% \mathrm{~B}$, at 11.0 min and to $90 \% \mathrm{~B}$ at 16.0 min Finally, $90 \% \mathrm{~B}$ was maintained for $1.0 \mathrm{~min}$ followed by column re-equilibration during $13.0 \mathrm{~min}$ under the initial conditions. The first $2.2 \mathrm{~min}$ and from min 17.0 until the end of the run, the diverter valve turned the effluent from 
the column into waste in order to prevent soiling the source in the MS detector. Flow rate was $0.4 \mathrm{~mL} / \mathrm{min}$. Injection volume was $15 \mu \mathrm{L}$.

Detection was achieved using a 6410 Triple Quad (QqQ) LC-MS/MS System from Agilent Technologies (Germany) equipped with an electrospray ionization (ESI) interface. Data acquisition parameters for selected reactions monitoring (SRM) mode were optimized for all mycotoxins, one by one, in flow injection analysis (FIA) mode. SCAN mode was used to choose the precursor ion. $[\mathrm{M}+\mathrm{H}]^{+},\left[\mathrm{M}+\mathrm{NH}_{4}\right]^{+}$and $[\mathrm{M}+\mathrm{Na}]^{+}$ adducts were searched. SIM mode was used to choose fragmentor voltage, and Product Ion mode was used to choose the product ions and appropriate collision energy for each one.

MS operation conditions were as follows: capillary voltage $4000 \mathrm{~V}$, drying and nebulizing gas (high purity nitrogen) at $350^{\circ} \mathrm{C}$, at a flow of $9 \mathrm{~L} / \mathrm{min}$ and at 40 psi. Ultra high purity nitrogen (99.999\% purity, Praxair, Spain) was used inside the collision cell. Data has been collected using SRM mode.

\subsection{Calibration sample preparation}

Calibration samples were prepared by spiking UHT cow milk with mycotoxins. Previously, UHT cow milk, in which trichothecenes were not be detected, was selected. A given volume of the mixed stock solution was pipetted into $15 \mathrm{~mL}$ polypropylene centrifuge tubes and dried under vacuum at $65^{\circ} \mathrm{C}$ in an evaporator (GeneVac, SP Scientific, England). The residue was then dissolved in $1 \mathrm{~mL}$ of UHT cow milk by stirring for $2 \mathrm{~min}$ in a vortex agitator. Next, the procedure for sample preparation was applied.

\subsection{Sample preparation}

Milk samples were prepared as follows: $1 \mathrm{~mL}$ of milk sample was extracted with $4 \mathrm{~mL}$ of acetonitrile acidified with formic acid (2\%) for $15 \mathrm{~min}$, using a $360^{\circ}$ rotary agitator. The mixture was centrifuged (10 $\mathrm{min}$ at $5000 \mathrm{rpm})$ and $4.1 \mathrm{~mL}$ of the clear extract, the maximum volume that can be securely separated, were transferred into a clean tube. Next, approximately $60 \mathrm{mg}$ of $\mathrm{CH}_{3} \mathrm{COONa}$ were added to the extract and after shaking for $15 \mathrm{~min}$ in a rotary agitator, the mixture was centrifuged for $5 \mathrm{~min}$ at $5000 \mathrm{rpm}$. A total of $3.5 \mathrm{~mL}$ of the upper extract (ACN phase) was transferred into a clean tube and 
evaporated at $65^{\circ} \mathrm{C}$ until dryness. The residue was reconstituted in $200 \mu \mathrm{L}$ of mobile phase (5\% B) by vortexing for 2 min Finally, this was filtered through a $0.45 \mu \mathrm{m}$ PVDF filter (Merck Millipore, Ireland) before LC-MS/MS analysis.

\subsection{Validation of the method}

Validation of the method for simultaneous quantification of trichothecenes in UHT cow milk has been based on the following parameters: selectivity, detection and quantification limits, range of concentration, linearity, precision of the instrumental system, precision and accuracy (within- and between-day), recovery, matrix effect, stability in the injector tray after extracting mycotoxins from UHT cow milk, stability in the mixed stock solution stored at $-20^{\circ} \mathrm{C}$, and stability in the dried extract obtained from spiked milk sample and stored at $-20^{\circ} \mathrm{C}$.

Selectivity was assessed using a mass spectrometer (QqQ) in SRM mode. Two transitions were selected for each one of the mycotoxins. The transitions intensity ratio (qualification/quantification) has been calculated in percentage for each mycotoxin at three concentration levels in both, matrix matched calibration samples and in standard samples. The mean value obtained for each mycotoxin in each type of sample has been included in table 1 . RSD values obtained in each type of sample at the three levels of concentration are below $10 \%$ in all cases and the difference between mean values in both types of samples in less than $12 \%$ for all the mycotoxins. These data, along with the comparison of the chromatographic retention times with those of standards (with a tolerance of $\pm 0.5 \%$ ), assured peak identity [32].

Quantification and detection limits were assessed for each one of the mycotoxins by preparing calibration samples at low concentrations. The limit of quantification (LOQ) corresponds to the minimum concentration assayed in UHT cow milk with adequate precision (RSD < 20\%) and accuracy (back-calculated concentration with a relative error (RE) compared to the nominal value $<20 \%$ ). This concentration level has been included at the lowest level in the corresponding calibration curve. The limit of detection (LOD) was the lowest concentration level tested without adequate precision and accuracy at which a value of signal-to-noise $(\mathrm{S} / \mathrm{N})$ ratio of at least 3 was obtained for the less sensitive transition. 
Range has been chosen for each one of the mycotoxins for covering from LOQ to $10 \mathrm{x}$ LOQ concentration levels.

Four calibration curves were plotted for each one of the mycotoxins on four different days. All the calibration curves were made up using 8 calibration points prepared by spiking milk samples, as indicated before. Matrix-matched calibration curves were evaluated by the analysis of determination coefficient $r^{2}>0.99$, the slope of the linear calibration curve statistically different from $0 \quad(p=95 \%)$ and back-calculated concentration for each one of the calibration samples (relative error $<15 \%$ or $<20 \%$ for LOQ level).

Precision of the instrument was calculated as peak areas RSD\% of six consecutive injections of one vial prepared from a calibration sample at medium level of concentration. Accuracy and precision were assayed by analyzing three calibration samples at low (3 x LOQ), medium (6 x LOQ) and high levels (10 x LOQ) of each one of the calibration ranges, per triplicate, on one day (within-run) and on four different days (between-run). Precision has been calculated as the relative standard deviation (RSD) in \%, whereas the standard error of the mean compared to the nominal value was used for accuracy.

Recovery and matrix effect were determined at low, medium and high levels of concentration per triplicate on three different days. In order to do this, for each concentration level, the adequate volume of the mixed stock solution of mycotoxins were put into nine tubes and after that, the solvent was evaporated. In three of the tubes (tubes A), the residue was dissolved in $1 \mathrm{~mL}$ of UHT cow milk and after that the sample was treated as indicated before. The residues in three other tubes (tubes B) were dissolved with $3.5 \mathrm{~mL}$ of final extract of a non-spiked milk sample, evaporated and resuspended in $200 \mu \mathrm{L}$ of mobile phase; and the residues in other three tubes (tubes $\mathrm{C}$ ) were directly dissolved in $200 \mu \mathrm{L}$ of mobile phase.

Recovery was obtained for each mycotoxin and concentration level as follows:

$$
R \%=\frac{\text { Mean of the peak areas from milk spiked before extraction (tubes A) }}{\text { Mean of the peak areas from milk spiked after extraction (tubes B) }} \times 100 \%
$$


Precision (between-run) of the recovery value obtained for each mycotoxin should be $\leq$ $15 \%$.

Matrix effect was obtained for each mycotoxin and concentration level using the following equation [33]:

$$
\% M E=\frac{\text { Mean of the peak areas from milk spiked after extraction(tubes } \mathrm{B})}{\text { Mean of the peak areas in mobile phase }(\text { tubes } \mathrm{C})} \times 100
$$

If $\% \mathrm{ME}=100$, no matrix effects are considered; if $\% \mathrm{ME}>100$, there is signal enhancement, and if \% ME $<100$, there is signal suppression.

Stability in the injector tray $\left(4^{\circ} \mathrm{C}\right)$ was assayed at three different concentrations (low, medium and high levels) in triplicate injected at 0,12 and $49 \mathrm{~h}$ after being prepared. In order to study the stability of the mycotoxins at $-20^{\circ} \mathrm{C}$, spiked milk samples at three concentration levels (low, medium and high levels) and in triplicate were extracted; after evaporation of the $\mathrm{ACN}$ phase, they were stored at $-20^{\circ} \mathrm{C}$. This procedure was repeated during four weeks. Finally (fourth week), all the residues were reconstituted with mobile phase and the peak areas obtained from frozen samples were compared with those obtained from fresh samples. Stability was evaluated by carrying out a regression study of the obtained peak areas for each mycotoxin versus time. A slope value statistically non-different from $0(p=95 \%)$ indicated stability.

In addition, stability of mycotoxins in the mixed stock solution at $-20^{\circ} \mathrm{C}$ was evaluated at one concentration level, analyzing a $100 \mu \mathrm{L}$ aliquot of this solution per triplicate, stored for 21 days at $-20^{\circ} \mathrm{C}$ and also analyzing one $100 \mu \mathrm{L}$ aliquot of a fresh mixed stock solution per triplicate. Aliquots were evaporated at $65^{\circ} \mathrm{C}$ and re-dissolved in 500 $\mu \mathrm{L}$ of mobile phase $(5 \% \mathrm{~B})$. Stability has been assured if the mean peak area for each mycotoxin, after being stored at $-20^{\circ} \mathrm{C}$, was a value among the mean peak areas $\pm 15 \%$ for each mycotoxin in the fresh mixed stock solution.

\section{Results and discussion}

3.1 Optimization of mass spectrometry and chromatography conditions 
ESI in positive and negative mode were explored, but better results were obtained in positive mode. Precursor and product ions, fragmentor, collision energy and retention time were set for each mycotoxin (Table 1). Most of precursor ion and transitions chosen concur with the reference literature $[34,35]$.

Different chromatographic conditions (100\% methanol or mixtures of ACN and methanol as solvent B in the mobile phase and different gradients) were investigated in order to achieve the best separation and resolution of peaks. Using the chromatographic conditions described, A (5mM ammonium formate and $0.1 \%$ formic acid in water): B (5mM ammonium formate and $0.1 \%$ formic acid in 95:5 methanol:water) in gradient conditions, adequate separations of the mycotoxin peaks were obtained. Five percent of water in the $\mathrm{B}$ component of mobile phase was necessary for preventing ammonium formate precipitation. A chromatogram obtained from a spiked milk sample at LOQ level is show in Fig. 1.

\subsection{Preparation of the mixed stock solution}

Different mixed stock solutions have been prepared for spiking milk samples. In order to evaluate them, an aliquot of each one has been evaporated, re-dissolved in mobile phase and analyzed in triplicate. In each case, mean peak areas from different mixed stock solutions did not differ in more than 5\% and therefore, it has been assumed that they were prepared correctly.

\subsection{Procedure for milk spiking}

Two spiking methods were tested in order to obtain calibration samples as similar as possible to the real samples: to dry the adequate volume of the mixed stock solution and afterwards dissolve the residue in $1 \mathrm{~mL}$ of milk by vortexing; or directly to add the volume of the mixed stock solution to $1 \mathrm{~mL}$ of milk. Higher peak areas and lesser RSD (\%) were obtained after applying the first method. Perhaps the addition of acetonitrile could alter the samples, for instance, protein precipitation or emulsion rupture, thereby creating additional difficulties in the extraction.

\subsection{Extraction procedure}

Milk is a complex matrix due to its high content in fat, protein and sugars, all of which interfere during the extraction of mycotoxins and during extract cleanup. Different 
solvents have been used for the extraction of mycotoxins from milk [36]. From all of these, acetonitrile, acetone, methanol, ethyl acetate and different mixtures of acetonitrile-acetone were chosen for testing their extraction capability. All of them were acidified with formic acid (2\%). In addition, the use of hexane (for removing fat) or different salts (sodium acetate, sodium chloride, or magnesium sulfate) were evaluated. All experiments were carried out in triplicate.

First of all, acetonitrile, acetone, methanol and ethyl acetate, acidified with formic acid (2\%), were evaluated mixing $4 \mathrm{~mL}$ of one of these solvents with $1 \mathrm{~mL}$ of spiked milk. After centrifugation (12 min at $5000 \mathrm{rpm}), 4 \mathrm{~mL}$ of the clear extract were evaporated at $65^{\circ} \mathrm{C}$ until dryness. The residue was reconstituted in $200 \mu \mathrm{L}$ of mobile phase (5\% B) by vortexing for $2 \mathrm{~min}$, filtered and analyzed. The peak area obtained for each mycotoxin when ACN was used was considered to represent $100 \%$ extraction and then the extraction of each mycotoxin was calculate in $\%$ of peak area in regard to that obtained using ACN (Fig. 2). As can be observed, acetonitrile and acetone give, on the average, the best extraction for all 10 trichothecenes tested (Fig. 2A). Therefore, different mixtures of acetonitrile-acetone (4:0, 3:1, 2:2, 1:3, 0:4) acidified with formic acid were assayed, but ACN gave the best results (Fig 2B). Finally, different proportions of ACN:milk (2:1, 3:1, 4:1, 6:1, 8:1) were assayed and the best results (the best efficient extraction procedure and less volume used) were obtained when $4 \mathrm{~mL}$ of $\mathrm{ACN}$ were added to $1 \mathrm{~mL}$ of milk (Fig. 2C).

Next, the use of hexane for removing fats was evaluated $[18,37]$. In order to do this, 1 $\mathrm{mL}$ of milk sample was extracted with $4 \mathrm{~mL}$ of $\mathrm{ACN}$ ( $2 \%$ formic acid) and $2 \mathrm{~mL}$ of hexane. It was observed that if hexane is added to milk before ACN, a gelatinous phase appears, making the work to be carried out more difficult. After stirring for $30 \mathrm{~min}$ and centrifugation, the ACN-water phase was separated, evaporated and re-suspended in $200 \mu \mathrm{L}$ of mobile phase (5\% B), followed by analysis. The use of hexane increases the extraction ratio of mycotoxin from milk to the $\mathrm{ACN}$-water phase, but creates additional difficulties in the sample extraction procedure and increases \%RSD.

In order to increase concentration, the extract from each sample was evaporated and resuspended in mobile phase before analysis. The evaporation process was timeconsuming because a yellow viscous pellet, containing undetermined components 
extracted from milk, appeared in the tube preventing complete dryness and adequate resuspension. In order to obtain a clean dry extract, and based on reference literature [38], the addition of certain salts during the extraction procedure, such as $\mathrm{NaCl}$ and $\mathrm{CH}_{3} \mathrm{COONa}$, with or without the simultaneous use of $\mathrm{MgSO}_{4}$, was studied. It was observed that the addition of $\mathrm{MgSO}_{4}$ formed a clump that could be trapping mycotoxins and $\mathrm{MgSO}_{4}$ was discontinued. The use of $60 \mathrm{mg}$ of $\mathrm{CH}_{3} \mathrm{COONa}$ with $\mathrm{ACN}$ as the extraction solution obtained the best results: a clear separation of the water phase and ACN was achieved and the acetonitrile phase could be totally evaporated without visible yellow residue in the tubes. Therefore, this clean-up procedure was chosen instead of using ACN and hexane. Sodium acetate should be added to the mixture obtained after ACN extraction, because the peak areas obtained for HT-2 and T-2 (the least polar mycotoxins of those tested) were considerably smaller when sodium acetate was added to the milk samples prior to extraction.

\subsection{Method validation}

Good calibration curves were obtained for each one of the mycotoxins because the regression study between the response (peak areas) and the respective concentration of mycotoxins in calibration samples met the previously fixed linearity criteria. Table 2 shows the following: linear ranges for each mycotoxin; the regression data for one of the regression lines obtained for each mycotoxin, which includes $r^{2}(>0.99)$, slope (along with their confidence intervals $(p=95 \%)$ that did not include the 0 value), intercept values and the LOD and LOQ (the slowest concentration level in each mycotoxin range) for each mycotoxin.

Developed methods for mycotoxins determination should have the maximum sensitivity possible. Different procedures have been used by different authors in order to define the lowest quantifiable concentration level for trichothecenes. LOQ values obtained in this work are better than those obtained by authors that use a similar procedure in their calculation (the lowest concentration level validated with satisfactory recovery and precision). Tsiplakou et al reported an LOQ of $10 \mu \mathrm{g} / \mathrm{kg}$ for DAS, T-2 and HT-2 [31]; and Beltrán et al reported values of $4 \mu \mathrm{g} / \mathrm{kg}$ for NEO, T-2 and DAS, $40 \mu \mathrm{g} / \mathrm{kg}$ for NIV, DON, 3-ADON, 15-ADON, FUS-X and HT-2, and $400 \mu \mathrm{g} / \mathrm{kg}$ for NIV [30]. Others determined the decision limit $(\mathrm{CC} \alpha)$ and the detection capability $(\mathrm{CC} \beta)$, for this reason 
results are difficult to be compared [39]. Jia et al [29] obtained CC $\beta$ values from 0.03 $\mu \mathrm{g} / \mathrm{kg}$ for DOM-1 up to $0.88 \mu \mathrm{g} / \mathrm{kg}$ for trichothecenes. Sørensen et al [18] obtained from $0.05 \mu \mathrm{g} / \mathrm{L}$ for DAS up to $0.15 \mu \mathrm{g} / \mathrm{L}$ for DON.

Instrumental precision was studied by analyzing one spiked milk sample six times at the medium level for each mycotoxin; a RSD (\%) below 6.5 was achieved for each one of them. Within-run $(n=3)$ and between-run $(n=12)$ precision (expressed as \%RSD) and accuracy (as the relative error of the mean of back-calculated concentrations with respect to the nominal value) were less than $15 \%$ in the three concentration levels assayed for all mycotoxins. Table 3 shows the results obtained in the precision and accuracy studies.

Recovery values (\%) obtained (and RSD \%) for each mycotoxin and concentration level in intermediate precision conditions $(n=3)$ are shown in Table 3. Mean recovery values (\%) taking into account different levels and days (n=9) were: 63.5, 73.1, 70.5, 74.7, 75.8, 73.9, 72.9, 73.4, 75.6, 72.3 for NIV, DON, DOM-1, FUS-X, NEO, 3-ADON, 15ADON, DAS, HT-2 and T-2, respectively, with RSD (\%) value $\leq 15 \%$ for all the mycotoxins. All of the results were within the performance criteria range for DON, T-2 and HT-2, established in the Commission Regulation (EC) N N $^{\circ}$ 401/2006 of February 23, 2006 which lays down the methods for sampling and analysis for the official control of the levels of mycotoxins in foodstuffs $\mathrm{DON}(>100 \leq 500 \mu \mathrm{g} / \mathrm{L})$ : recovery between 60 $110 \%, \mathrm{RSD}_{\mathrm{r}} \leq 20 \%$ and $\mathrm{RSD}_{\mathrm{R}} \leq 40 \%$; $\mathrm{T}-2(50-250 \mu \mathrm{g} / \mathrm{L})$ : recovery between $60-130 \%$, $\mathrm{RSD}_{\mathrm{r}} \leq 40 \%$ and a $\mathrm{RSD}_{\mathrm{R}} \leq 60 \%$; HT-2 (100-200 $\left.\mu \mathrm{g} / \mathrm{L}\right)$ : recovery between $60-130 \%$, $\mathrm{RSD}_{\mathrm{r}} \leq 40 \%$ and a $\mathrm{RSD}_{\mathrm{R}} \leq 60 \%[40]$.

Matrix Effect was evaluated at low, medium and high levels as indicated in the material and methods section. Table 3 shows \%ME values obtained (and RSD \%) for each mycotoxin and concentration level in intermediate precision conditions $(n=3)$. Significant signal suppression or enhancement was observed for most of them, with DON being the most affected. Mean \%ME taking into account different levels and days $(n=9)$ were: $66.3,22.1,72.8,83.4,120.7,90.5,81.1,110.7,107.6,58.2$ for NIV, DON, DOM-1, FUS-X, NEO, 3-ADON, 15-ADON, DAS, HT-2 and T-2, respectively, with RSD $(\%) \leq 15 \%$, except for T-2 (24.3\%). 
Stability studies

Prepared samples were stable inside the injector $\left(4^{\circ} \mathrm{C}\right)$ for at least $49 \mathrm{~h}$; extracted and evaporated samples were stable at $-20^{\circ} \mathrm{C}$ for at least four weeks for all mycotoxins except for FUS-X, which was stable for two weeks. Mixed stock solution is stable at $20^{\circ} \mathrm{C}$ for at least 21 days.

\subsection{Applicability of proposed method}

Thirteen whole UHT cow milk samples packaged in Tetra Brick from different brands were purchased in Navarra (Spain) and analyzed using the proposed method that has proven to be an excellent tool in the screening, quantitation and confirmation of target mycotoxins in UHT cow milk. Mycotoxin levels higher than LOD have not been found in any one of the samples. An example of the chromatogram obtained from a real sample is shown in Fig. 1

\section{Conclusions}

In order to determine the co-occurrence of mycotoxins in animal milk in a shorter period of time and with a lower cost of analysis, new analytical methodologies, which make simultaneous analysis in different matrices possible, must be developed. In this paper, LC-ESI-MS/MS has been applied for the simultaneous analysis of 10 trichothecenes in UHT cow milk. The method includes an extraction step using acidified ACN, and sodium acetate in the cleanup of the extract to eliminate components from the matrix. The proposed method has been successfully validated for its implantation in routine monitoring programs. The analyzed samples did not present trichothecenes, but the analysis of more samples from different countries is needed in order to obtain a conclusion regarding the presence of these mycotoxins in milk and its significance on milk safety.

\section{Acknowledgements}

We wish to thank the "Programa de Investigación Universidad de Navarra" (PIUNA) for financial support. M. Flores Flores wishes to express her gratitude to the "Asociación de Amigos de la Universidad de Navarra" (ADA) for the grant funding. 
Wish to extend our gratitude to the reviewers for their comments and suggestions which have served to enhanced the quality of the manuscript.

\section{References:}

[1] Binder EM. Managing the risk of mycotoxins in modern feed production. Anim Feed Sci Technol 133 (2007) 149-166. 10.1016/j.anifeedsci.2006.08.008.

[2] Joint Research Centre - European Commission. European Union Reference Laboratories (EURL)-Mycotoxins. 2014; Available at: https://ec.europa.eu/jrc/en/eurl/mycotoxins. Accessed May 29th, 2015.

[3] Wu F, Groopman JD, Pestka JJ. Public health impacts of foodborne mycotoxins. Annu Rev Food Sci Technol 5 (2014) 351-372. 10.1146/annurev-food-030713-092431.

[4] Hussein HS, Brasel JM. Toxicity, metabolism, and impact of mycotoxins on humans and animals. Toxicology 167 (2001) 101-134. 10.1016/S0300-483X(01)00471-1.

[5] Creppy EE. Update of survey, regulation and toxic effects of mycotoxins in Europe. Toxicol Lett 127 (2002) 19-28. 10.1016/S0378-4274(01)00479-9.

[6] Cavret S, Lecoeur S. Fusariotoxin transfer in animal. Food and Chemical Toxicology 44 (2006) 444-453. 10.1016/j.fct.2005.08.021.

[7] Broekaert N, Devreese M, De Baere S, De Backer P, Croubels S. Modified Fusarium mycotoxins unmasked: From occurrence in cereals to animal and human excretion. Food and Chemical Toxicology 80 (2015) 17-31. 10.1016/j.fct.2015.02.015.

[8] Placinta CM, D'Mello JPF, Macdonald AMC. A review of worldwide contamination of cereal grains and animal feed with Fusarium mycotoxins. Anim Feed Sci Technol 78 (1999) 21-37. 10.1016/S0377-8401(98)00278-8.

[9] Sun Y, Zhang G, Zhao H, Zheng J, Hu F, Fang B. Liquid chromatography-tandem mass spectrometry method for toxicokinetics, tissue distribution, and excretion studies of T-2 toxin and its major metabolites in pigs. Journal of Chromatography B 958 (2014) 75-82. 10.1016/j.jchromb.2014.03.010. 
[10] European Food Safety Authority. Scientific Opinion on the risks for animal and public health related to the presence of T-2 and HT-2 toxin in food and feed. EFSA Panel on Contaminants in the Food Chain (CONTAM). Parma, Italy. EFSA Journal 9 (2011) 2481. Available at: http://www.efsa.europa.eu/en/efsajournal/doc/2481.pdf.

[11] Capriotti AL, Caruso G, Cavaliere C, Foglia P, Samperi R, Lagana A. Multiclass mycotoxin analysis in food, environmental and biological matrices with chromatography/mass spectrometry. Mass Spectrom Rev 31 (2012) 466-503. 10.1002/mas.20351.

[12] Mbandi E, Pestka JJ. Deoxynivalenol and satratoxin G potentiate proinflammatory cytokine and macrophage inhibitory protein 2 induction by Listeria and Salmonella in the macrophage. J Food Prot 69 (2006) 1334-1339.

[13] European Food Safety Authority. Scientific Opinion on risks for animal and public health related to the presence of nivalenol in food and feed. EFSA Journal 11 (2013) 3262.

[14] Joint FAO/WHO Expert Committee on Food Additives (JECFA). Seventy-second meeting Rome, 16-25 February 2010, Summary and conclusions. Issued 16th March 2010. JECFA/72/SC (2010) Available at: http://www.who.int/foodsafety/chem/summary72_rev.pdf.

[15] Food and Agriculture Organization (FAO). Milk and milk products. 2015; Available at: $\quad$ http://www.fao.org/agriculture/dairy-gateway/milk-and-milkproducts/en/\#.VW3IBc tmkp. Accessed May 29th, 2015.

[16] European Commission. Milk and milk products. 2015; Available at: http://ec.europa.eu/agriculture/milk/index_en.htm. Accessed May 29th, 2015.

[17] Fink-Gremmels J. Mycotoxins in cattle feeds and carry-over to dairy milk: a review. Food Addit Contam Part A 25 (2008) 172-180. 10.1080/02652030701823142.

[18] Sørensen LK, Elbæk TH. Determination of mycotoxins in bovine milk by liquid chromatography tandem mass spectrometry. J Chromatogr B Analyt Technol Biomed Life Sci 820 (2005) 183-196. 10.1016/j.jchromb.2005.03.020. 
[19] European Commission. Commission Recommendation of 17 August 2006 on the presence of deoxynivalenol, zearalenone, ochartoxin A, T-2 and HT-2 and fumonisins in products intended for animal feeding (2006/576/EC). OJ L 229 (2006) 7-9.

[20] Côté LM, Dahlem AM, Yoshizawa T, Swanson SP, Buck WB. Excretion of deoxynivalenol and its metabolite in milk, urine, and feces of lactating dairy cows. $\mathbf{J}$ Dairy Sci 69 (1986) 2416-2423. 10.3168/jds.S0022-0302(86)80681-6.

[21] Seeling K, Danicke S, Valenta H, Van Egmond HP, Schothorst RC, Jekel AA, Lebzien P, Schollenberger M, Razzazi-Fazeli E, Flachowsky G. Effects of Fusarium toxin-contaminated wheat and feed intake level on the biotransformation and carry-over of deoxynivalenol in dairy cows. Food Addit Contam 23 (2006) 1008-1020. $10.1080 / 02652030600723245$.

[22] Keese C, Meyer U, Valenta H, Schollenberger M, Starke A, Weber IA, Rehage J, Breves G, Danicke S. No carry over of unmetabolised deoxynivalenol in milk of dairy cows fed high concentrate proportions. Mol Nutr Food Res 52 (2008) 1514-1529. 10.1002/mnfr.200800077.

[23] Robison TS, Mirocha CJ, Kurtz HJ, Behrens JC, Chi MS, Weaver GA, Nystrom SD. Transmission of T-2 toxin into bovine and porcine milk. J Dairy Sci 62 (1979) 637641. 10.3168/jds.S0022-0302(79)83301-9.

[24] Yoshizawa T, Mirocha CJ, Behrens JC, Swanson SP. Metabolic fate of T-2 toxin in a lactating cow. Food Cosmet Toxicol 19 (1981) 31-39. 10.1016/0015-6264(81)90300$\mathrm{X}$.

[25] Mirocha CJ, Pathre SV, Robison TS. Comparative metabolism of zearalenone and transmission into bovine milk. Food Cosmet Toxicol 19 (1981) 25-30. 10.1016/00156264(81)90299-6.

[26] Valimaa AL, Kivisto AT, Leskinen PI, Karp MT. A novel biosensor for the detection of zearalenone family mycotoxins in milk. J Microbiol Methods 80 (2010) 4448. 10.1016/j.mimet.2009.10.017. 
[27] Flores-Flores ME, Lizarraga E, López de Cerain A, González-Peñas E. Presence of mycotoxins in animal milk: A review. Food Control 53 (2015) 163-176. 10.1016/j.foodcont.2015.01.020.

[28] FAO. Worldwide regulations for mycotoxins in food and feed in 2003. Food and Agriculture Organization of The United Nations. FAO Food and Nutrition Paper 81 (2004).

[29] Jia W, Chu X, Ling Y, Huang J, Chang J. Multi-mycotoxin analysis in dairy products by liquid chromatography coupled to quadrupole orbitrap mass spectrometry. Journal of Chromatography A 1345 (2014) 107-114. 10.1016/j.chroma.2014.04.021.

[30] Beltrán E, Ibáñez M, Portolés T, Ripollés C, Sancho JV, Yusà V, Marín S, Hernández F. Development of sensitive and rapid analytical methodology for food analysis of 18 mycotoxins included in a total diet study. Anal Chim Acta 783 (2013) 3948. 10.1016/j.aca.2013.04.043.

[31] Tsiplakou E, Anagnostopoulos C, Liapis K, Haroutounian SA, Zervas G. Determination of mycotoxins in feedstuffs and ruminant's milk using an easy and simple LC-MS/MS multiresidue method. Talanta 130 (2014) 8-19. 10.1016/j.talanta.2014.06.018.

[32] European Commission. Commission Decision of 12 August 2002 implementing Council Directive 96/23/EC concerning the performance of analytical methods and the interpretation of results (2002/657/EC). OJ L 221 (2002) 8-36.

[33] Gosetti F, Mazzucco E, Zampieri D, Gennaro MC. Signal suppression/enhancement in high-performance liquid chromatography tandem mass spectrometry. Journal of Chromatography A; Mass Spectrometry: Innovation and Application.Part VI 1217 (2010) 3929-3937. 10.1016/j.chroma.2009.11.060.

[34] Capriotti AL, Cavaliere C, Foglia P, Samperi R, Stampachiacchiere S, Ventura S, Laganà A. Multiclass analysis of mycotoxins in biscuits by high performance liquid chromatography-tandem mass spectrometry. Comparison of different extraction procedures. Journal of Chromatography A $1343 \quad$ (2014) 69-78. 10.1016/j.chroma.2014.04.009. 
[35] Koesukwiwat U, Sanguankaew K, Leepipatpiboon N. Evaluation of a modified QuEChERS method for analysis of mycotoxins in rice. Food Chem 153 (2014) 44-51. j.foodchem.2013.12.029.

[36] Mol HG, Plaza-Bolanos P, Zomer P, de Rijk TC, Stolker AA, Mulder PP. Toward a generic extraction method for simultaneous determination of pesticides, mycotoxins, plant toxins, and veterinary drugs in feed and food matrixes. Anal Chem 80 (2008) 9450-9459. 10.1021/ac801557f.

[37] Food and Agriculture Organization (FAO). Mycotoxin prevention and control in foodgrains. Overview of analytical methods for mycotoxin contamination in maize and peanuts. Available at: http://www.fao.org/docrep/x5036e/x5036E0c.htm. Accessed May 26th, 2015.

[38] Rubert J, Leon N, Saez C, Martins CP, Godula M, Yusa V, Manes J, Soriano JM, Soler C. Evaluation of mycotoxins and their metabolites in human breast milk using liquid chromatography coupled to high resolution mass spectrometry. Anal Chim Acta 820 (2014) 39-46. 10.1016/j.aca.2014.02.009.

[39] Van Loco J, Jànosi A, Impens S, Fraselle S, Cornet V, Degroodt JM. Calculation of the decision limit $(\mathrm{CC} \alpha)$ and the detection capability $(\mathrm{CC} \beta)$ for banned substances: The imperfect marriage between the quantitative and the qualitative criteria. Anal Chim Acta 586 (2007) 8-12. 10.1016/j.aca.2006.11.058.

[40] European Commission. Commission Regulation (EC) No 401/2006 of 23 February 2006 laying down the methods of sampling and analysis for the official control of the levels of mycotoxins in foodstuffs. OJ L 70 (2006) 12 


\section{Figure captions:}

Fig. 1: Superposed extracted SRM for each one of the mycotoxins in one chromatogram. (A) Includes the quantification transition peaks obtained after extraction of a spiked milk sample at LOQ level. (B) Shows the qualification transition peaks obtained from the same sample. (C) Shows the quantification transition peaks obtained from a real milk sample purchased from the market
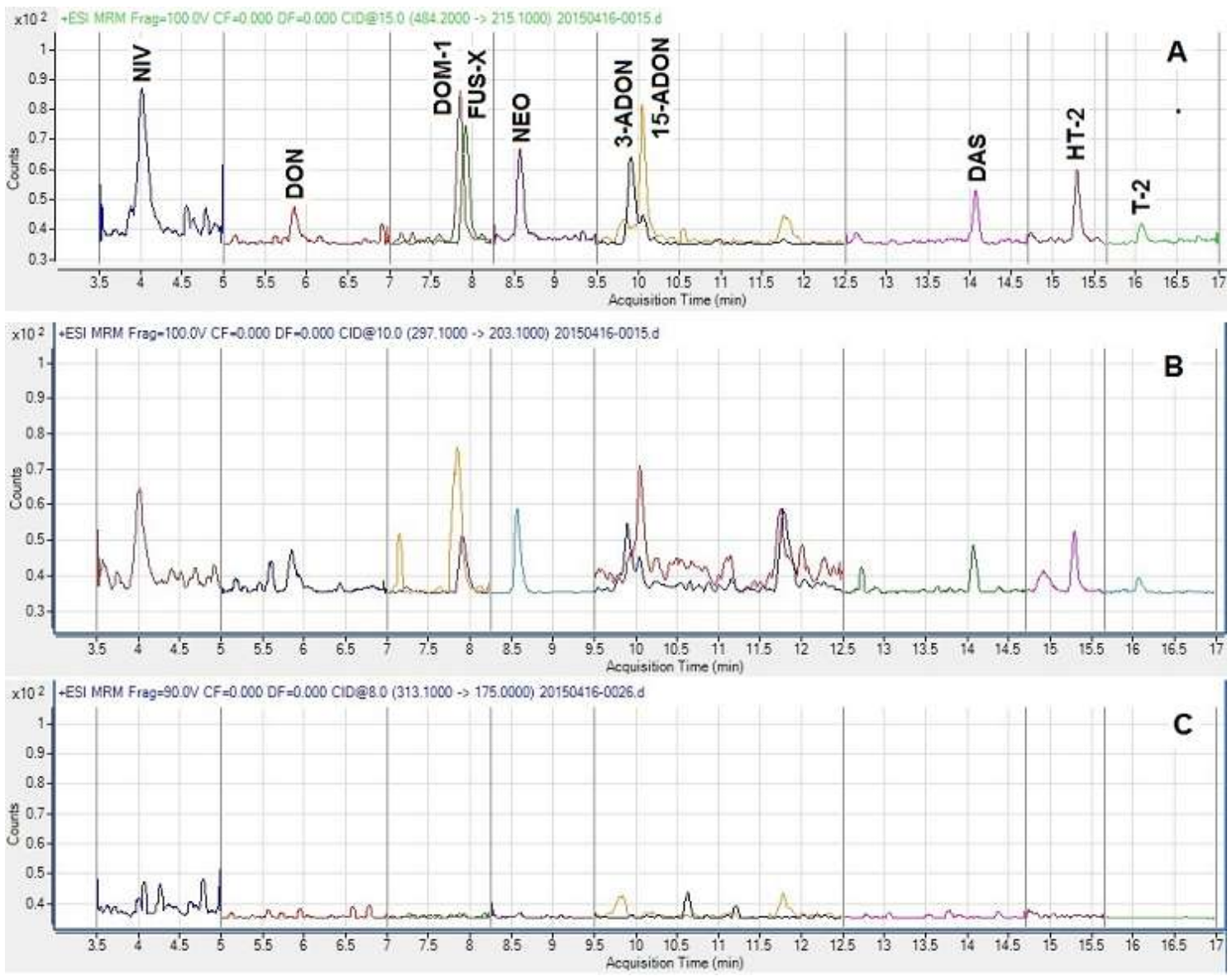
Fig 2. Mycotoxin extraction with different solvents (data normalized versus ACN) (A), with different acetonitrile:acetone proportions (data normalized versus ACN:acetone (4:0)) (B) and different proportions of extraction mixture (ACN):milk (data normalized versus (4:1)) (C) .
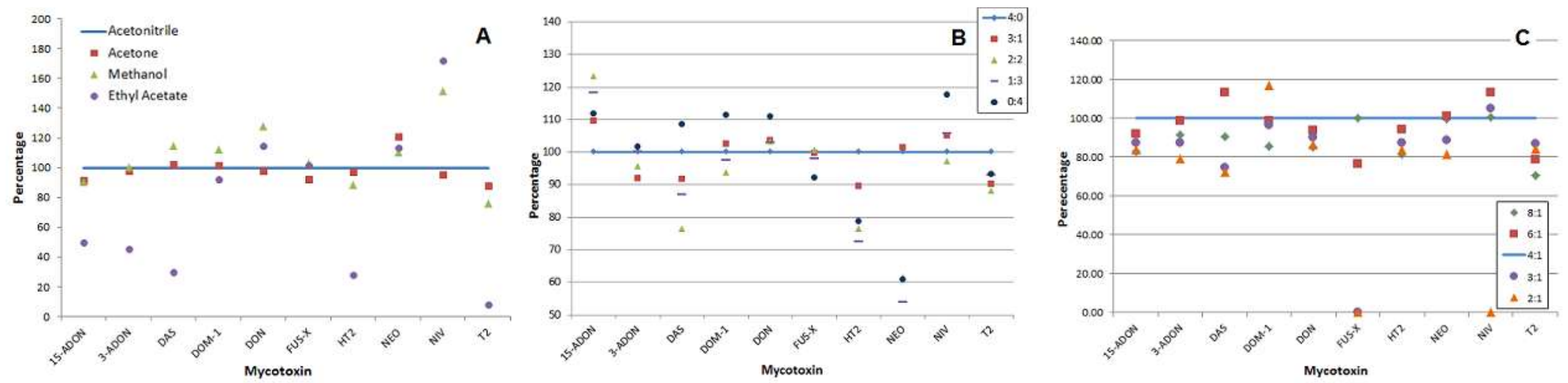
Table 1: Retention times and MS parameters for identifying each mycotoxin.

\begin{tabular}{cccccccccc}
\hline $\begin{array}{c}\mathbf{t}_{\boldsymbol{R}}{ }^{\mathbf{a}} \\
(\mathbf{m i n})\end{array}$ & Mycotoxin & Adduct & $\begin{array}{c}\text { Precursor } \\
\text { Ion }\end{array}$ & $\begin{array}{c}\text { Product } \\
\text { Ion }(\mathbf{Q})^{\mathbf{a}}\end{array}$ & $\begin{array}{c}\text { Product } \\
\text { Ion }(\mathbf{q})^{\mathbf{a}}\end{array}$ & $\begin{array}{c}\text { Frag. }^{\text {a }} \\
(\mathbf{V})\end{array}$ & $\mathbf{C E}^{\mathbf{a}}(\mathbf{V})$ & $\begin{array}{c}\text { Relative } \\
\text { intensity \% } \\
(\mathbf{s a m p l e})\end{array}$ & $\begin{array}{c}\text { Relative } \\
\text { intensity \% } \\
\text { (standard) }\end{array}$ \\
\hline 4.0 & $\mathrm{NIV}$ & $\mathrm{H}^{+}$ & 313.1 & 175.0 & 177.1 & 90 & $8 / 10^{\mathrm{b}}$ & 60 & 62 \\
5.9 & $\mathrm{DON}$ & $\mathrm{H}^{+}$ & 297.1 & 249.1 & 203.1 & 100 & $3 / 10$ & 92 & 93 \\
7.8 & $\mathrm{DOM}-1$ & $\mathrm{H}^{+}$ & 281.2 & 215.2 & 233.1 & 90 & $5 / 5$ & 96 & 92 \\
7.9 & $\mathrm{FUS}-\mathrm{X}$ & $\mathrm{H}^{+}$ & 355.1 & 175.0 & 247.1 & 80 & $22 / 8$ & 56 & 57 \\
8.6 & $\mathrm{NEO}$ & $\mathrm{NH}_{4}{ }^{+}$ & 400.1 & 185.1 & 305.2 & 100 & $15 / 5$ & 65 & 57 \\
9.9 & $3-\mathrm{ADON}$ & $\mathrm{H}^{+}$ & 339.2 & 231.1 & 203.2 & 90 & $3 / 7$ & 70 & 69 \\
10.0 & $15-\mathrm{ADON}$ & $\mathrm{H}^{+}$ & 339.2 & 137.1 & 321.2 & 90 & $3 / 0$ & 81 & 78 \\
14.1 & $\mathrm{DAS}$ & $\mathrm{NH}_{4}{ }^{+}$ & 384.2 & 247.1 & 307.1 & 90 & $7 / 5$ & 79 & 85 \\
15.3 & $\mathrm{HT}-2$ & $\mathrm{NH}_{4}{ }^{+}$ & 442.2 & 215.1 & 197.1 & 90 & $7 / 10$ & 66 & 64 \\
16.1 & $\mathrm{~T}-2$ & $\mathrm{NH}_{4}{ }^{+}$ & 484.2 & 215.1 & 305.1 & 100 & $15 / 7$ & 61 & 57 \\
\hline
\end{tabular}

${ }^{a} t_{R}$, retention time; Q, quantification; q, qualification; Frag, Fragmentor; CE, Collision energy.

${ }^{b}$ First value corresponds to (Q) and second value to (q). Relative intensity calculated as q/Q x100, mean of values obtained at three concentrations.

Table 2: Linear range, LOQ (as the lowest level of the range), LOD, signal-to-noise ratio for LOD and regression data for a typical calibration curve

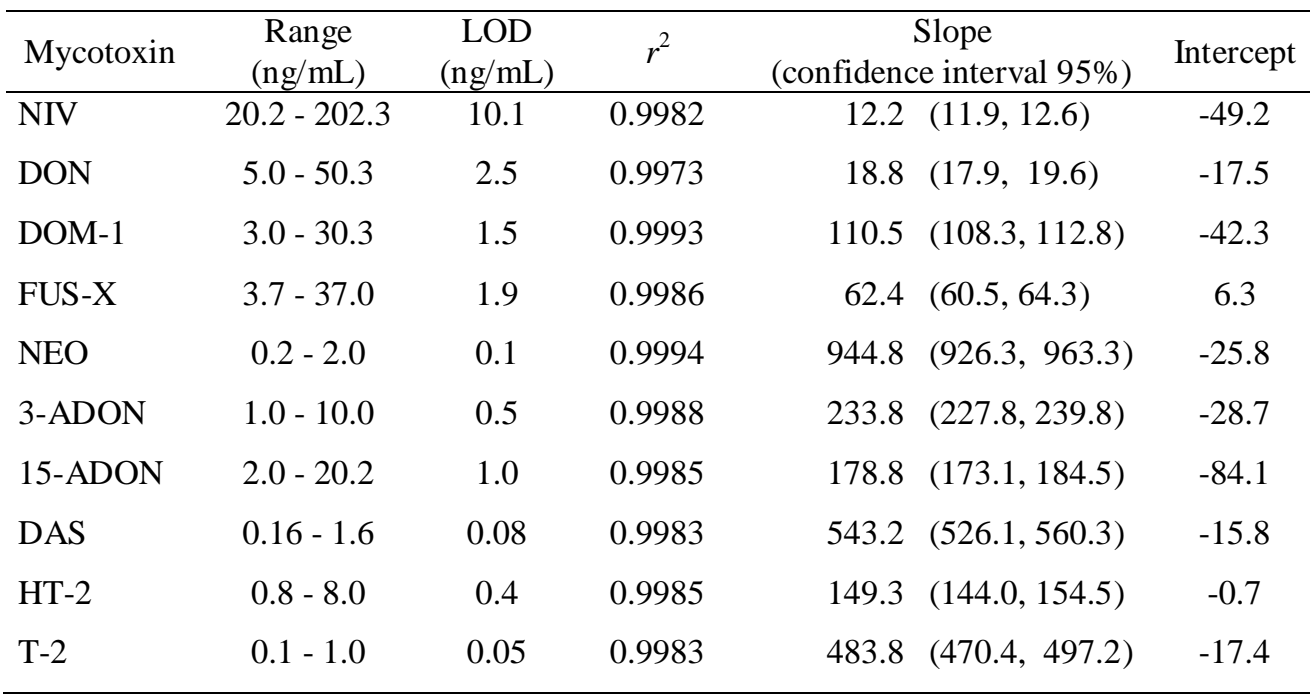


Table 3: Precision $(\% \mathrm{RSD})$, accuracy $(\% \mathrm{E})$, matrix effect $(\% \mathrm{ME})$ and recovery $(\% \mathrm{R})$. Within-run precision and accuracy have been studied in triplicate at each concentration level, whereas between-run have been studied in triplicate on four different days. ME and recovery have been studied in between-run conditions on three days.

\begin{tabular}{|c|c|c|c|c|c|c|c|c|c|c|c|c|c|c|c|c|c|c|c|c|c|c|c|c|}
\hline \multirow[t]{3}{*}{ Mycotoxin } & \multicolumn{6}{|c|}{ Precision (\%RSD) } & \multicolumn{6}{|c|}{ Accuracy $(\% \mathrm{E})$} & \multirow{2}{*}{\multicolumn{6}{|c|}{$\begin{array}{c}\text { Matrix effect (\%RSD) } \\
\text { Between-run }(n=3)\end{array}$}} & \multirow{2}{*}{\multicolumn{6}{|c|}{$\begin{array}{l}\text { Recovery (\%RSD) } \\
\text { Between-run (n=3) }\end{array}$}} \\
\hline & \multicolumn{3}{|c|}{ Within-run $(n=3)$} & \multicolumn{3}{|c|}{ Between-run ( $\mathrm{n}=12)$} & \multicolumn{3}{|c|}{ Within-run $(n=3)$} & \multicolumn{3}{|c|}{ Between-run $(n=12)$} & & & & & & & & & & & & \\
\hline & $\mathrm{La}$ & M & $\mathrm{H}$ & $\mathrm{L}$ & M & $\mathrm{H}$ & $\mathrm{L}$ & M & $\mathrm{H}$ & $\mathrm{L}$ & M & $\mathrm{H}$ & $\mathrm{L}$ & & M & & $\mathrm{H}$ & & $\mathrm{L}$ & & M & & $\mathrm{H}$ & \\
\hline NIV & 8.5 & 3.9 & 0.5 & 7.3 & 6.9 & 6.3 & 5.9 & 2.3 & 0.8 & 6.0 & 2.9 & 3.6 & 65.8 & $(12.7)$ & 67.5 & $(13.5)$ & 65.6 & $(16.6)$ & 61.7 & $(7.4)$ & 62.0 & (8.3) & 66.7 & $(6.9)$ \\
\hline DON & 13.6 & 5.3 & 3.0 & 8.5 & 11.2 & 4.2 & 10.1 & 6.8 & 2.1 & 2.4 & 2.8 & 3.1 & 22.6 & $(13.6)$ & 22.5 & $(15.2)$ & 21.1 & $(15.4)$ & 72.6 & (8.8) & 68.6 & (1.9) & 78.3 & (4.8) \\
\hline DOM-1 & 4.8 & 3.2 & 1.2 & 3.9 & 10.1 & 5.4 & 3.5 & 1.2 & 0.1 & 3.1 & 0.7 & 1.0 & 75.6 & (8.3) & 71.9 & $(4.0)$ & 71.0 & $(5.3)$ & 64.8 & $(8.9)$ & 68.1 & (4.1) & 78.6 & (9.0) \\
\hline FUS-X & 2.0 & 4.3 & 3.9 & 9.6 & 5.8 & 4.1 & 4.4 & 0.1 & 3.9 & 4.8 & 1.9 & 3.1 & 88.0 & (4.8) & 82.0 & $(2.6)$ & 80.3 & (5.2) & 75.0 & $(12.5)$ & 69.8 & (2.8) & 79.5 & (3.5) \\
\hline NEO & 2.0 & 3.6 & 1.5 & 4.4 & 11.5 & 6.3 & 2.6 & 1.2 & 1.4 & 4.3 & 3.3 & 1.4 & 120.9 & (5.4) & 118.3 & $(0.8)$ & 122.8 & $(3.4)$ & 75.9 & $(3.2)$ & 72.6 & (3.1) & 79.0 & (3.7) \\
\hline 3-ADON & 4.5 & 1.1 & 3.2 & 6.2 & 8.8 & 4.3 & 0.5 & 3.0 & 2.9 & 2.2 & 2.0 & 0.8 & 99.8 & (6.6) & 86.5 & $(3.1)$ & 85.2 & $(2.5)$ & 67.2 & (12.3) & 74.7 & (6.7) & 79.7 & (4.9) \\
\hline $15-\mathrm{ADON}$ & 0.1 & 6.3 & 0.9 & 5.7 & 11.1 & 2.6 & 5.3 & 3.5 & 3.1 & 0.3 & 3.1 & 0.5 & 86.8 & $(14.0)$ & 79.1 & $(3.9)$ & 77.5 & $(5.9)$ & 67.6 & $(8.5)$ & 74.1 & (4.7) & 76.8 & (3.2) \\
\hline DAS & 9.6 & 8.0 & 1.0 & 9.5 & 12.4 & 3.3 & 0.2 & 6.9 & 3.5 & 1.3 & 9.2 & 0.3 & 121.4 & $(3.4)$ & 106.4 & (7.9) & 104.1 & $(6.6)$ & 69.3 & (7.4) & 72.8 & $(8.2)$ & 78.2 & $(8.2)$ \\
\hline HT-2 & 8.6 & 2.8 & 5.1 & 6.0 & 12.8 & 4.4 & 1.8 & 5.8 & 5.8 & 2.1 & 7.7 & 1.8 & 109.2 & (4.7) & 104.5 & $(5.7)$ & 109.1 & (11.2) & 75.6 & $(2.6)$ & 72.2 & (11.5) & 79.2 & (5.1) \\
\hline $\mathrm{T}-2$ & 0.9 & 10.1 & 3.8 & 8.8 & 15.5 & 13.2 & 1.7 & 6.9 & 2.2 & 2.0 & 6.0 & 1.4 & 53.3 & (2.0) & 59.6 & (31.7) & 61.8 & (31.8) & 71.2 & (14.0) & 64.5 & (18.0) & 81.1 & (6.8) \\
\hline
\end{tabular}

${ }^{\mathrm{a}} \mathrm{L}, \mathrm{M}, \mathrm{H}$ : low (3 x LOQ), medium (6 x LOQ) and high levels (10 x LOQ), of each mycotoxin, respectively. 


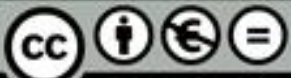

BY NC ND Esta obra está bajo una licencia de Creative Commons Reconocimiento-

NoComercial-SinObraDerivada 4.0 Internacional. 